\title{
Infrared-faint radio sources are at high redshifts
}

\section{Spectroscopic redshift determination of infrared-faint radio sources using the Very Large Telescope}

\author{
A. Herzog ${ }^{1,2,3}$, E. Middelberg ${ }^{1}$, R. P. Norris ${ }^{3,4}$, R. Sharp ${ }^{5}$, L. R. Spitler ${ }^{2,6}$, and Q. A. Parker ${ }^{2,6}$ \\ 1 Astronomisches Institut, Ruhr-Universität Bochum, Universitätsstr. 150, 44801 Bochum, Germany \\ e-mail: herzog@astro.rub.de \\ 2 Macquarie University, Sydney, NSW 2109 Sydney, Australia \\ 3 CSIRO Astronomy and Space Science, Marsfield, PO Box 76, Epping, NSW 1710 Sydney, Australia \\ 4 ARC Centre of Excellence for All-sky Astrophysics (CAASTRO), Australia \\ ${ }^{5}$ Research School of Astronomy \& Astrophysics, Australian National University, Mount Stromlo Observatory, Cotter road, \\ Weston Creek, ACT 2611 Camberra, Australia \\ 6 Australian Astronomical Observatory, PO Box 915, NSW 1670 North Ryde, Australia
}

Received 30 November 2013 / Accepted 16 June 2014

\section{ABSTRACT}

\begin{abstract}
Context. Infrared-faint radio sources (IFRS) are characterised by relatively high radio flux densities and associated faint or even absent infrared and optical counterparts. The resulting extremely high radio-to-infrared flux density ratios up to several thousands were previously known only for high-redshift radio galaxies (HzRGs), suggesting a link between the two classes of object. However, the optical and infrared faintness of IFRS makes their study difficult. Prior to this work, no redshift was known for any IFRS in the Australia Telescope Large Area Survey (ATLAS) fields which would help to put IFRS in the context of other classes of object, especially of HzRGs.

Aims. This work aims at measuring the first redshifts of IFRS in the ATLAS fields. Furthermore, we test the hypothesis that IFRS are similar to HzRGs, that they are higher-redshift or dust-obscured versions of these massive galaxies.

Methods. A sample of IFRS was spectroscopically observed using the Focal Reducer and Low Dispersion Spectrograph 2 (FORS2) at the Very Large Telescope (VLT). The data were calibrated based on the Image Reduction and Analysis Facility (IRAF) and redshifts extracted from the final spectra, where possible. This information was then used to calculate rest-frame luminosities, and to perform the first spectral energy distribution modelling of IFRS based on redshifts.

Results. We found redshifts of $1.84,2.13$, and 2.76, for three IFRS, confirming the suggested high-redshift character of this class of object. These redshifts and the resulting luminosities show IFRS to be similar to HzRGs, supporting our hypothesis. We found further evidence that fainter IFRS are at even higher redshifts.

Conclusions. Considering the similarities between IFRS and HzRGs substantiated in this work, the detection of IFRS, which have a significantly higher sky density than HzRGs, increases the number of active galactic nuclei in the early universe and adds to the problems of explaining the formation of supermassive black holes shortly after the Big Bang.
\end{abstract}

Key words. techniques: spectroscopic - galaxies: active - galaxies: distances and redshifts - galaxies: high-redshift

\section{Introduction}

Active galactic nuclei (AGN) at high redshifts are an important field of current research since they play a crucial role in answering basic questions about the evolution of the Universe. For example, high-redshift AGN are used to study the evolution of the link between black hole mass and the properties of its host galaxy (e.g. Hopkins et al. 2005; Lamastra et al. 2010), their impact on the reionisation and the structure formation in the universe (e.g. Fan et al. 2006; Robertson et al. 2010; Boutsia et al. 2011), and the growth of supermassive black holes (SMBHs) with masses $>10^{9} M_{\odot}$ in less than one billion years after the Big Bang (e.g. Volonteri \& Rees 2005). Infrared-faint radio sources - a class of object whose detection was unexpected - could significantly contribute to the population of high-redshift AGN.

\subsection{Definition and discovery of IFRS}

Infrared-faint radio sources (IFRS) are peculiar objects, characterised by relatively high radio flux densities on the order of $1 \mathrm{mJy}$ at $1.4 \mathrm{GHz}$ only associated with faint or even absent infrared (IR) counterparts. They are defined by Zinn et al. (2011) by two criteria:

(i) radio-to-IR flux density ratio $S_{1.4 \mathrm{GHz}} / S_{3.6 \mu \mathrm{m}}>500$ and (ii) $3.6 \mu \mathrm{m}$ flux density $S_{3.6 \mu \mathrm{m}}<30 \mu \mathrm{Jy}$.

The first criterion selects objects that are clear outliers from the radio-IR correlation, while the second criterion prevents the selection of low-redshift objects with $z \lesssim 1.4$ (Zinn et al. 2011).

The discovery of IFRS was unexpected. When Norris et al. (2006) crossmatched the deep $1.4 \mathrm{GHz}$ radio maps from the Australia Telescope Large Area Survey (ATLAS) with the data from the Spitzer Wide-area Infrared Extragalactic Survey (SWIRE; Lonsdale et al. 2003), it was expected that SWIRE would provide an IR counterpart for any extragalactic radio source detected in ATLAS. The spectral energy distribution (SED) of typical host galaxies at redshifts below 4, regardless of whether the radio emission is dominated by AGN or star forming activity, should place the IR flux above the 
SWIRE detection limit (Norris et al. 2006). However, 22 sources were found in the Chandra Deep Field South (CDFS) without an IR counterpart in the crossmatching process. These sources - constrained at $3.6 \mu \mathrm{m}$ by a $5 \sigma$ upper limit of $5 \mu \mathrm{Jy}-$ were labelled as IFRS. Using the same approach, Middelberg et al. (2008a) identified 31 IFRS using ATLAS radio observations of the European Large Area IR space observatory Survey South 1 (ELAIS-S1) field and SWIRE data. In total, 53 IFRS were detected in the overlapping region between SWIRE and ATLAS, with $1.4 \mathrm{GHz}$ flux densities between tenths and tens of mJy. Zinn et al. (2011) noted that none of those IFRS has an X-ray counterpart in the XMM-Newton survey of the ELAIS-S1 field (Puccetti et al. 2006). One IFRS is located in the field of the CDFS Chandra 2 Ms survey (Giacconi et al. 2002; Luo et al. 2008; minimum full band sensitivity around $3.3 \times$ $10^{-16} \mathrm{erg} \mathrm{s}^{-1} \mathrm{~cm}^{-2}$ over the Great Observatories Origins Deep Survey-South field). However, this IFRS also remains undetected in the X-ray regime.

\subsection{VLBI observations and SED modelling}

The first attempts to further characterise the properties of IFRS used Very Long Baseline Interferometry (VLBI) observations. Norris et al. (2007) observed two IFRS and identified an AGN in one of them, showing a linear size of $\leq 260 \mathrm{pc}$ at any redshift. Furthermore, Middelberg et al. (2008b) detected one of four targeted IFRS with VLBI and derived a brightness temperature of $3.6 \times 10^{6} \mathrm{~K}$, implying non-thermal emission from an AGN since thermal emission processes cannot produce such high brightness temperatures. Therefore, Norris et al. and Middelberg et al. concluded that at least a fraction of all IFRS contain AGN.

Garn \& Alexander (2008) found a sample of 14 IFRS in the Spitzer First Look Survey (FLS) field, using Spitzer Infrared Array Camera (IRAC; Fazio et al. 2004) and Multiband Imaging Photometer (MIPS; Rieke et al. 2004) data. They concluded from SED modelling that IFRS are probably 3C sources redshifted to $2 \leq z \leq 5$. Moreover, they excluded obscured star forming galaxies (SFGs) as an explanation for the objects in their sample because of the radio-to-IR flux density ratio upper limits, differing significantly from corresponding values of SFGs.

The first SED modelling of ATLAS-IFRS was presented by Huynh et al. (2010), using new ultra-deep imaging in the extended CDFS (eCDFS). From their detailed SED modelling of four IFRS, Huynh et al. concluded that a 3C 273-like object can reproduce the data when redshifted to $z>2$. In agreement with Garn \& Alexander (2008), Huynh et al. showed that all four analysed IFRS fall well beyond the radio-IR correlation, suggesting the radio emission is produced by the presence of an AGN, but not by star forming activity.

\subsection{Similarities between IFRS and HzRGs}

Middelberg et al. (2011) studied the radio properties of 17 out of all 31 IFRS from the ATLAS ELAIS-S1 sample between $2.3 \mathrm{GHz}$ and $8.4 \mathrm{GHz}$. They found a median radio spectral index of $\alpha=-1.4^{1}$ and no index larger than -0.7 , which is significantly steeper than the radio spectra of the general source population $(-0.86)$ and of the AGN source population $(-0.82)$ in the ATLAS ELAIS-S1 field. Furthermore, Middelberg et al. noticed similarities between IFRS and the sample of high-redshift radio galaxies (HzRGs) from Seymour et al. (2007). These

The spectral index is defined as $S \propto v^{\alpha}$.
HzRGs show steep radio spectra (median radio spectral index of -1.02) like IFRS. Moreover, the extremely high radio-to-IR flux density ratios of IFRS overlap with those of this HzRG sample. Recently, Singh et al. (2014) searched for HzRGs in faint ultra steep spectrum (USS) radio sources and found several sources overlapping with the selection criteria of IFRS.

The hypothesis of an AGN existing in at least a fraction of all IFRS was supported by Middelberg et al. who identified ten IFRS of their sample as AGN and the other seven as probable AGN, based on radio-to-IR flux density rations, polarisation properties, radio spectral indices, VLBI detections, or radio morphology.

These similarities between IFRS and HzRGs were emphasised by Norris et al. (2011) who showed that no other type of object occupies the range of radio-to-IR flux density ratios of IFRS except for HzRGs. The sample of HzRGs of Seymour et al. (2007) shows a relation between the $3.6 \mu \mathrm{m}$ flux density and redshift similar to the $K-z$ relation for other radio galaxies (Willott et al. 2003). Norris et al. suggested that IFRS might follow the same correlation. They used deep data from the Spitzer Extragalactic Representative Volume Survey (SERVS; Mauduit et al. 2012) with a $3 \sigma$ noise level of $\sim 1.5 \mu \mathrm{Jy}$ at $3.6 \mu \mathrm{m}$ in the fields of CDFS and ELAIS-S1 and stacked the $3.6 \mu \mathrm{m}$ images of 39 IFRS, which resulted in an upper flux density limit of $\sim 0.2 \mu \mathrm{Jy}$, but remained without a detection. This was interpreted as evidence for the significant IR faintness of IFRS.

\subsection{IFRS catalogues}

Zinn et al. (2011) compiled a catalogue of 55 IFRS in four deep radio fields, CDFS, ELAIS-S1, FLS, and Cosmological Evolution Survey (COSMOS, Schinnerer et al. 2007), based on their criteria mentioned in Sect. 1.1 and derived a surveyindependent IFRS sky density of $(30.8 \pm 15.0) \mathrm{deg}^{-2}$.

Recently, Maini et al. (in prep.) found 21 IFRS in the Lockman Hole and looked for IR counterparts of IFRS located in the SERVS deep fields. Collier et al. (2014) used data from the all-sky survey Wide-Field Infrared Survey Explorer (WISE; Wright et al. 2010) and the Unified Radio Catalog (URC; Kimball \& Ivezić 2008) and presented a catalogue of 1317 IFRS that fulfill the selection criteria from Zinn et al. (2011) given in Sect. 1.1 but are, on average, brighter than the IFRS found in the ATLAS fields. Collier et al. suggested that their IFRS are closer versions of the IFRS found by Norris et al. (2006) and Middelberg et al. (2008a).

We note that IFRS were originally selected as radio sources without any IR counterpart (i.e. Norris et al. 2006; Middelberg et al. 2008a; Garn \& Alexander 2008). Since this definition was survey-specific, Zinn et al. (2011) generalised this criterion to the two survey-independent selection criteria given in Sect. 1.1. However, these two types of selection criteria are very similar in the sense that they select the same class of object. The definition by Norris et al. allows faint radio sources that are slightly above the noise level and show no counterpart at $3.6 \mu \mathrm{m}$ to be considered as an IFRS, whereas this is prevented by the Zinn et al. criteria. On the other hand, Zinn et al. consider objects with a faint near-IR counterpart as an IFRS, although only under the condition that the source is sufficiently radio-bright to fulfill the radio-to-IR flux density ratio criterion.

\subsection{Current status of research}

All observational findings so far are compatible with most, if not all, IFRS being high-redshift $(z>2)$ radio-loud AGN, 
Table 1. Sample of spectroscopically observed IFRS.

\begin{tabular}{lcccccccc}
\hline \hline $\begin{array}{l}\text { IFRS } \\
\text { ID }\end{array}$ & RA & Dec & $\begin{array}{c}S_{1.4 \mathrm{GHz}} \\
{[\mathrm{mJy}]}\end{array}$ & $\begin{array}{c}S_{3.6 \mu \mathrm{m}} \\
{[\mu \mathrm{Jy}]}\end{array}$ & $S_{1.4 \mathrm{GHz}} / S_{3.6 \mu \mathrm{m}}$ & $\begin{array}{c}R(\mathrm{Vega}) \\
{[\mathrm{mag}]}\end{array}$ & $\begin{array}{c}t_{\text {obs }} \\
{[\mathrm{min}]}\end{array}$ & $z$ \\
\hline S212 & $03: 29: 48.942$ & $-27: 31: 48.98$ & 18.9 & 17.5 & 1080 & 22.0 & 44 & $2.76 \pm 0.05$ \\
S265 & $03: 30: 34.661$ & $-28: 27: 06.51$ & 18.6 & 29.3 & 635 & 22.3 & 88 & $1.84 \pm 0.03$ \\
S539 & $03: 33: 30.542$ & $-28: 54: 28.22$ & 9.1 & 14.2 & 641 & 24.1 & 45 & - \\
S713 & $03: 35: 37.525$ & $-27: 50: 57.88$ & 16.4 & 25.5 & 643 & 22.4 & 128 & $2.13 \pm 0.03$ \\
\hline
\end{tabular}

Notes. Listed are the ID, the position in RA and Dec, the radio flux density at $1.4 \mathrm{GHz}$, the IR flux density at $3.6 \mu \mathrm{m}$, the radio-to-IR flux density ratio between $1.4 \mathrm{GHz}$ and $3.6 \mu \mathrm{m}$, the optical $R$ band (Vega) magnitude, the on-source time, and the redshift determined in this work. Positions, flux densities, and magnitudes were taken from Norris et al. (2006).

potentially suffering from heavy dust extinction. Considering their high sky density, IFRS could be a very numerous and so far overlooked population of high-redshift AGN that could have a significant impact on the evolution of the universe as suggested by Zinn et al. (2011). Furthermore, Zinn et al. concluded that the $\mathrm{X}$-ray emission of AGN-driven IFRS is consistent with the unresolved components of the Cosmic X-ray background reported by Moretti et al. (2003).

The current knowledge about IFRS is wholly based on photometric detections or upper limits, apart from the VLBI observations. In this paper, we present the first spectroscopic data of IFRS in the ATLAS fields. Based on these observations made using the Focal Reducer and low dispersion Spectrograph 2 (FORS2; Appenzeller et al. 1998) on the Very Large Telescope (VLT), we present the first redshifts of ATLAS-IFRS. Using these results we test the hypothesis that IFRS are similar to HzRGs, derive their intrinsic properties, compare them to other objects and model the SEDs of IFRS. The sample of observed IFRS and the VLT FORS2 observations are described in Sect. 2. In Sect. 3, we summarise the data reduction and calibration and show the final spectra. In Sect. 4, redshifts are measured and intrinsic properties derived for our sample of IFRS. We test our hypotheses in Sect. 5 and present our conclusions in Sect. 6. The cosmological parameters used in this paper are $\Omega_{\Lambda}=0.7, \Omega_{\mathrm{M}}=0.3, H_{0}=70 \mathrm{~km} \mathrm{~s}^{-1} \mathrm{Mpc}^{-1}$ in combination with the calculator by Wright (2006).

\section{Sample and observations}

The sample of IFRS consists of four objects, chosen by their detected optical counterparts between 22.0 and 24.1 (Vega) magnitudes in the $R$ band. The condition of an optical counterpart ensures that the sources are bright enough for optical spectroscopy. All selected sources show a faint IR counterpart at $3.6 \mu \mathrm{m}$ between $14.2 \mu \mathrm{Jy}$ and $29.3 \mu \mathrm{Jy}$, fulfilling the selection criteria of IFRS by Zinn et al. (2011). The presence of an IR counterpart biases the observed sample towards less extreme IFRS, i.e. with lower radio-to-IR flux density ratios. In the case of the four observed IFRS, this ratio is between 600 and 1100 , whilst for the majority of ATLAS-IFRS, which do not show a detected IR counterpart, lower limits of this ratio can be as high as 8000 . These IR-undetected IFRS do not show an optical counterpart and, therefore, are not suitable for optical spectroscopy. Although the observed sample is not representative of all IFRS, it allows us to gather information about the intrinsic properties of less extreme IFRS and to test the hypotheses about their relation to other classes of object. Moreover, these findings will also allow conclusions on the more extreme IFRS which cannot be achieved by spectroscopy because of their deep optical and IR faintness. We summarise the observed objects and their characteristic properties in Table 1.

The longslit spectroscopic observations of these four IFRS were carried out in project 087.B-0813(A) between July and September 2011 (ESO Period 87) in service mode, using FORS2 on UT1 at the VLT. Using the GRISM_150I grism, covering the wavelength range between $330 \mathrm{~nm}$ and $1100 \mathrm{~nm}$, and a slit width of 2 ", a high throughput is achieved at the cost of relatively low resolution, although sufficient to provide reliable redshifts. The dispersion was $3.45 \AA$ /pixel, resulting in a resolution of $54.76 \AA$. The total on-source times were $44 \mathrm{~min}$ for $\mathrm{S} 212,88 \mathrm{~min}$ for $\mathrm{S} 265,45 \mathrm{~min}$ for S539, and $128 \mathrm{~min}$ for S713, where the exposure time for each object was split into several shorter exposures, enabling the correction of cosmic ray events. The seeing varied during and between the different observations from $0.86^{\prime \prime}$ to $2.46^{\prime \prime}$.

\section{Data reduction and calibration}

Data reduction was carried out using the standard Image Reduction and Analysis Facility (IRAF; Tody 1986) procedures. All exposures were bias-corrected and flatfielded, using normalised masterflats based on the individual dome flat exposures, whose faulty pixels were previously corrected using a bad pixel mask.

We rejected some single flat field exposures where an imperfect illumination was obvious before creating the masterflat. Cosmic rays were removed using the IRAF task cosmicrays and through manual inspection. Since the objects are not located in exactly one line in the two-dimensional spectra, i.e. the spatial position changes as a function of wavelength, we corrected the spatial axis for this distortion. Consequently, the wavelength calibration was carried out using lamp exposures taken in every observing night. Since all lamp exposures were saturated and the determination of the lines' peak positions was impossible, we applied a block average with width 3 on the spectral axis to all exposures used in the entire reduction procedure. At the end of the wavelength calibration, the data cover the wavelength range between $390 \mathrm{~nm}$ and $1100 \mathrm{~nm}$. Consequently, we subtracted the sky background using the IRAF task background. Finally, all individual exposures of one object were averaged to increase the signal-to-noise ratio and one-dimensional spectra were extracted by applying a suitable aperture at the object's position using the IRAF task apall. For IFRS S265, which was observed on 2011 July 16 and 2011 July 30, we used only the data taken on 2011 July 30 for the final spectrum because of the poor quality of the data taken on 2011 July 16, arising from seeing in the range of $2.5^{\prime \prime}$ compared to $0.9^{\prime \prime}$ on the other day. 

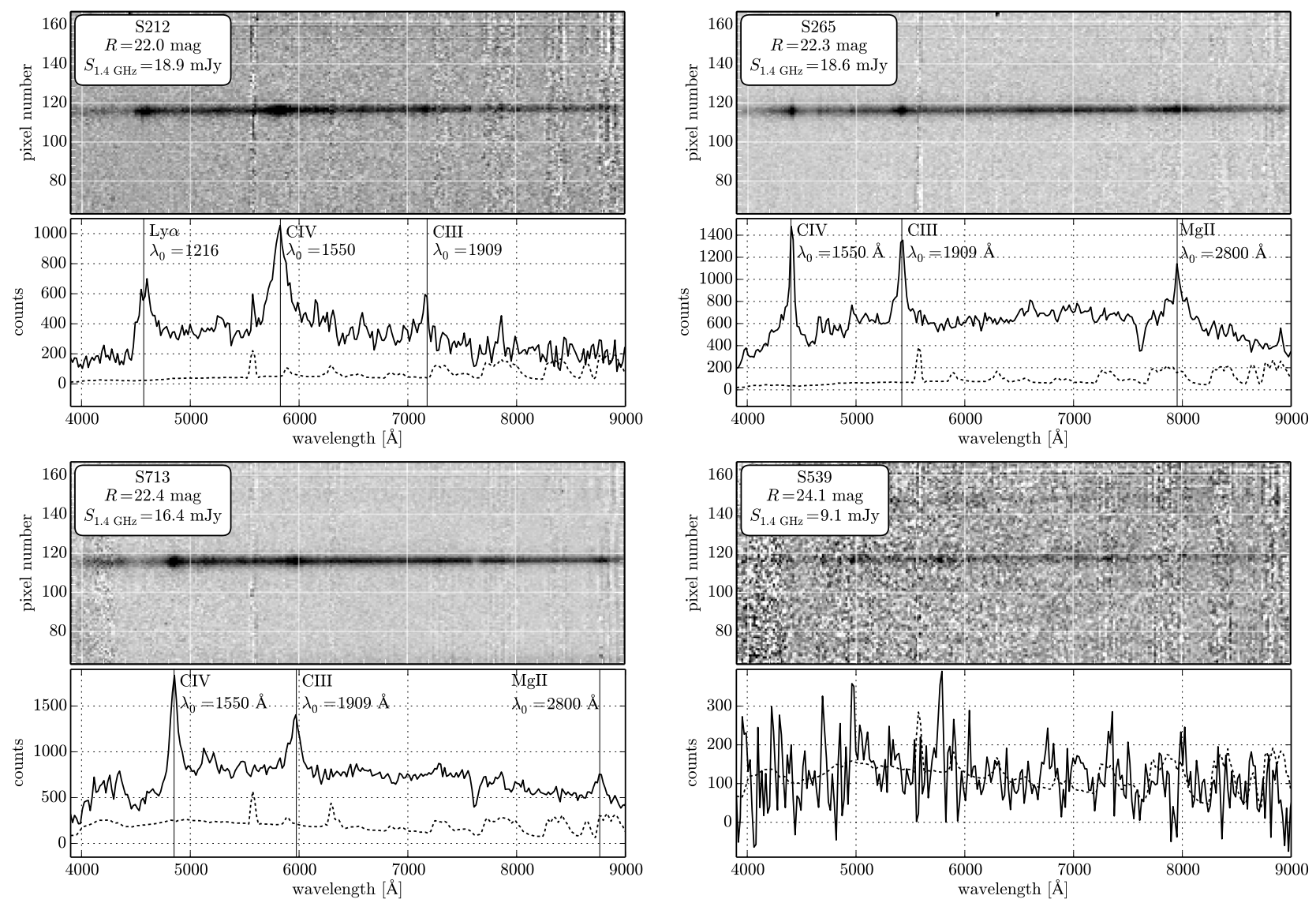

Fig. 1. Spectra of IFRS S212 (upper left), S265 (upper right), S713 (lower left), and S539 (lower right). For each IFRS we show the twodimensional spectrum (upper plot) and the extracted one-dimensional spectrum (lower plot). We list the ID, the $R$ band Vega magnitude, and the $1.4 \mathrm{GHz}$ radio flux density of each IFRS. In the one-dimensional spectrum, the solid line represents the spectrum of the IFRS while the dotted line shows the sky background in arbitrary units. Additionally, the position of emission lines are marked by vertical lines for a redshift of $z=2.76$ $(\mathrm{S} 212), z=1.84(\mathrm{~S} 265)$, and $z=2.13$ (S713).

The resulting one- and two-dimensional spectra of IFRS S212, S265, S713, and S539 are shown in Fig. 1.

\section{Redshifts and intrinsic properties of IFRS}

Using the one-dimensional spectra, we measured redshifts where applicable - from the Mg II line, which is generally considered to be the most reliable high ionisation line. Where Mg II was not available, we used C IV to derive the redshift, although $\mathrm{C}$ IV is known to be different from the galaxy's redshift (Richards et al. 2011). However, the effect is below one percent and we therefore do not observe this effect in our low-resolution spectra. The Ly $\alpha$ emission line is again less suitable for redshift determinations because of uncertainties due to self-absorption.

Since the calibration uncertainties in the spectra are negligible compared to the uncertainties in the determination of the line position, we determined the redshift uncertainties only from the error in the line position which was obtained from a Gaussian fit to the emission line. We note that all redshifts measured from all available emission lines listed in Table 2 are within the uncertainty of our best redshift measured from $\mathrm{Mg}$ II or C IV.

Using the obtained redshifts, we calculated K-corrected radio luminosities, assuming a power law $S \sim v^{\alpha}$ with a radio spectral index of -1.4 which is the median spectral index found by Middelberg et al. (2011) for a sample of 17 IFRS from the ATLAS sample. We also used the flattest $(-0.7)$ and steepest $(-2.4)$ spectral index from Middelberg et al. to constrain the expected radio luminosity range of the observed IFRS.
Table 2. Spectroscopic information of the four IFRS observed with FORS2.

\begin{tabular}{lccc}
\hline \hline $\begin{array}{l}\text { IFRS } \\
\text { ID }\end{array}$ & $z$ & Line & $\begin{array}{c}F W H M \\
{\left[\mathrm{~km} \mathrm{~s}^{-1}\right]}\end{array}$ \\
\hline S212 & $2.76 \pm 0.05$ & C IV & $8700 \pm 600$ \\
& $2.75 \pm 0.02$ & C III $]$ & $3300 \pm 700$ \\
S265 & $1.85 \pm 0.02$ & C IV & $3900 \pm 500$ \\
& $1.84 \pm 0.02$ & C III $]$ & $4200 \pm 300$ \\
& $1.84 \pm 0.03$ & Mg II & $8400 \pm 900$ \\
S713 & $2.13 \pm 0.03$ & C IV & $5400 \pm 700$ \\
& $2.12 \pm 0.03$ & C III $]$ & $5900 \pm 400$ \\
& $2.13 \pm 0.03$ & Mg II & $4600 \pm 1000$ \\
S539 & - & - & - \\
\hline
\end{tabular}

Notes. Listed are the IFRS ID, the spectrosopic redshift, and the emission line identified in the spectrum with the associated line width. We obtain the final redshift of each IFRS from the Mg II emission line and from C IV where Mg II was not available.

Hereafter, we state these numbers in brackets. In the following, we describe the individual spectra of our four observed IFRS, S212, S265, S713, and S539, respectively.

\section{1. $S 212$}

Three broad emission lines with full width at half maximum (FWHM) between $78 \AA$ and $169 \AA$ are visible in the spectrum of S212 which we identify as Ly $\alpha, \mathrm{CIV}$, and C III], at a 
redshift $z=2.76 \pm 0.05$ (see Fig. 1, upper left). Furthermore, a less distinct emission line can be associated with Si IV at around $5260 \AA$. The broad emission lines of a few thousand $\mathrm{km} \mathrm{s}^{-1}$ suggest the presence of an AGN in S212. Using the determined redshift and the measured radio flux density listed in Table $1, \mathrm{~S} 212$ has a $1.4 \mathrm{GHz}$ luminosity of $2.0 \times 10^{27} \mathrm{~W} \mathrm{~Hz}^{-1}$ $\left(8.8 \times 10^{26}-7.6 \times 10^{27} \mathrm{~W} \mathrm{~Hz}^{-1}\right)$.

\section{2. $S 265$}

We found three broad emission lines with FWHM between $57 \AA$ and $223 \AA$ in the spectrum of S265 in Fig. 1 (upper right) which we identify as C IV, C III], and $\mathrm{Mg}$ II, at a redshift $z=$ $1.84 \pm 0.03$. These broad emission lines with line widths in the range of $4000 \mathrm{~km} \mathrm{~s}^{-1}$ to $8000 \mathrm{~km} \mathrm{~s}^{-1}$ clearly suggest the presence of an AGN in $\mathrm{S} 265$. We find a $1.4 \mathrm{GHz}$ luminosity of $6.7 \times 10^{26} \mathrm{~W} \mathrm{~Hz}^{-1}\left(3.2 \times 10^{26}-1.2 \times 10^{27} \mathrm{~W} \mathrm{~Hz}^{-1}\right)$.

\section{3. $S 713$}

Three broad emission lines are visible in the spectrum of S713, with FWHMs between $884 \AA$ and $134 \AA$, corresponding to line widths about $5000 \mathrm{~km} \mathrm{~s}^{-1}$ and suggesting the presence of an AGN. The lines are associated with C IV, C III], and Mg II at a redshift $z=2.13 \pm 0.03$ (see Fig. 1, lower left). We find a $1.4 \mathrm{GHz}$ luminosity of $8.7 \times 10^{26} \mathrm{~W} \mathrm{~Hz}^{-1}\left(3.9 \times 10^{26}-2.7 \times 10^{27} \mathrm{~W} \mathrm{~Hz}^{-1}\right)$.

\section{4. $\$ 539$}

The source S539 is the optically faintest IFRS in this observing programme. Unfortunately, it was only observed with $44 \mathrm{~min}$ on-source time, although $3 \mathrm{~h}$ had been requested. Therefore, the resulting spectrum does not provide the quality of the other spectra and we cannot use it to measure a redshift and line widths (see Fig. 1, lower right). Despite the short integration time, emission features are visible, although with poor signal-to-noise. We tentatively interpret the emission feature at around $4970 \AA$ as Ly $\alpha$, indicated by the related break towards lower wavelengths, and a second emission feature at redder wavelengths as Si IV. We suggest S539 to be at redshift $z \sim 3.1$, although the low signalto-noise ratio prevents a reliable determination.

\section{Discussion}

We have obtained for the first time spectroscopic redshifts for three out of four IFRS in the ATLAS fields selected on the basis of their existing optical counterparts. All spectra provide solid determinations of redshifts between 1.8 and 2.8, providing strong evidence that these sources are located at high redshifts. The fourth IFRS has a low signal-to-noise ratio spectrum that indicates a redshift of 3.1 but needs additional confirmation. These redshifts are in agreement with the conclusions of Garn \& Alexander (2008), Huynh et al. (2010), Norris et al. (2011), and Zinn et al. (2011) who suggested that IFRS are located at redshifts above 2, mainly from SED modelling. As mentioned in Sect. 2, we are aware that the selection of the optically brightest IFRS might bias our sample towards lower redshifts. This seems to be in agreement with the measured redshifts, which are in the lower part of the expected redshift range of IFRS. Recently, Collier et al. (2014) presented 19 IFRS with spectroscopic redshifts from archival data. Their sample was extracted from a shallow all-sky survey, in contrast to the IFRS analysed in this paper which were found in the deep ATLAS fields. Therefore, the IFRS found by Collier et al. were radio brighter and slightly IR brighter than the ones presented in this work. Nevertheless, Collier et al. found redshifts in the range $2<z<3$, in agreement with those presented here.

All spectra shown in Fig. 1 are broad-line quasar spectra, characterised by high-ionisation emission lines with high equivalent widths. This finding agrees with former suggestions by Garn \& Alexander (2008), Huynh et al. (2010), Norris et al. (2011), and Zinn et al. (2011) that IFRS contain AGN.

\subsection{Similarity between IFRS and HzRGs}

In Sect. 4, we derived $1.4 \mathrm{GHz}$ radio luminosities between $6.7 \times$ $10^{26} \mathrm{~W} \mathrm{~Hz}{ }^{-1}$ and $2.0 \times 10^{27} \mathrm{~W} \mathrm{~Hz}^{-1}\left(3.2 \times 10^{26}-7.6 \times\right.$ $10^{27} \mathrm{~W} \mathrm{~Hz}^{-1}$ ) for the IFRS investigated in this work. The classical separation between Fanaroff \& Riley (1974) types 1 and 2 is $4.8 \times 10^{25} \mathrm{~W} \mathrm{~Hz}^{-1}\left(1.7 \times 10^{25} \mathrm{~W} \mathrm{~Hz}^{-1}\right)$ at $1.4 \mathrm{GHz}$, using a steep (ultrasteep) radio spectral index $\alpha=-0.8(-1.3)$ for the conversion from the $178 \mathrm{MHz}$ luminosity given by Fanaroff \& Riley. This clearly classifies IFRS as Fanaroff \& Riley type 2.

Seymour et al. (2007) defined an HzRG as a radio galaxy with $z>1$ and a $3 \mathrm{GHz}$ luminosity above $10^{26} \mathrm{~W} \mathrm{~Hz}^{-1}$, corresponding to $1.8 \times 10^{26} \mathrm{~W} \mathrm{~Hz}^{-1}\left(2.7 \times 10^{26} \mathrm{~W} \mathrm{~Hz}^{-1}\right)$ at $1.4 \mathrm{GHz}$. We find the IFRS in agreement with the radio luminosity range of HzRGs for the entire range of spectral indices of IFRS found by Middelberg et al. (2011), supporting the hypothesis of Norris et al. (2011) that IFRS might be siblings of HzRGs. We note that most IFRS are radio-brighter and possibly more radio-luminous than those analysed in this work.

Infrared-faint radio sources are characterised by their extremely high radio-to-IR flux density ratios typically in the range of several hundreds to a few thousands. The redshifts determined in Sect. 4 enable us to put these three IFRS in the plot showing the radio-to-IR flux density versus redshift (Fig. 2). This plot clearly separates IFRS from other classes of object which are typically found at high redshifts and indicates a potential connection between IFRS and HzRGs.

Based on this finding, Norris et al. (2011) suggested that IFRS might follow a relation between redshift and $3.6 \mu \mathrm{m}$ flux density, similar to the correlation found for the sample of HzRGs by Seymour et al. (2007). We can now test this hypothesis.

Figure 3 shows the $3.6 \mu \mathrm{m}$ IR flux density versus redshift for the three IFRS whose redshifts we determined in Sect. 4 and for the HzRGs from Seymour et al. (2007). Our IFRS clearly fall in the parameter space of HzRGs, supporting the hypothesis of Norris et al. in the tested redshift range between 1.8 and 2.8. This result provides evidence that the correlation works to redshifts of 2 or 3 . It seems that IFRS define the lower bound of HzRGs in this plot.

\subsection{Redshift-based SED modelling}

The availability of redshifts for IFRS now allows us to perform a more accurate SED modelling. Garn \& Alexander (2008), Huynh et al. (2010), and Zinn et al. (2011) modelled SEDs to constrain the redshift of IFRS.

Here, in contrast, we can use the redshift as an anchor and use it to test different SED templates against available photometric data. The method of our SED modelling is similar to the approaches by Huynh et al. (2010) and Zinn et al. (2011).

We built the SED templates using photometric data and redshifts from the NASA/IPAC Extragalactic Database (NED), connecting the datapoints by lines and smoothing the template. We used a variety of SED templates, including starburst, radio and 


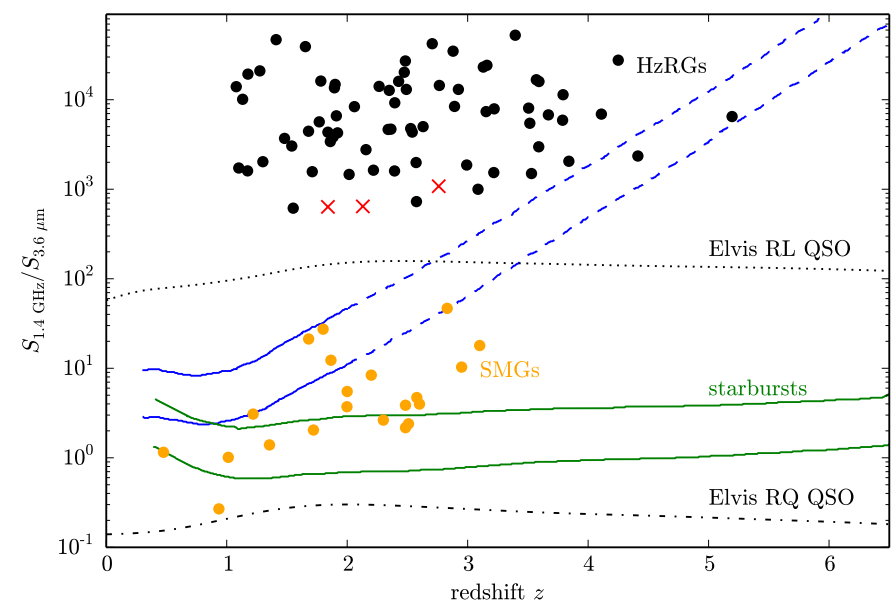

Fig. 2. Ratio of 1.4 GHz and $3.6 \mu \mathrm{m}$ flux densities for IFRS and several other classes of object as a function of redshift, adapted from Norris et al. (2011). It shows that the IFRS analysed in this paper (red crosses) are more similar to HzRGs (black dots, Seymour et al. 2007) than to other types of galaxies frequently found at high redshifts. The solid lines indicate the expected loci of luminous and ultra-luminous infrared galaxies (ULIRGs), using the templates from Rieke et al. (2009). The dotted and dot-dashed lines indicate the loci of a classical radio-loud and radio-quiet QSO, respectively, from Elvis et al. (1994). The location of classical submillimetre galaxies is indicated by the orange dots. We note that dust extinction could cause any of the calculated tracks to rise steeply at high redshift, where the observed $3.6 \mu \mathrm{m}$ emission is generated in visible wavelengths in the galaxy rest frame. This is illustrated by the dashed lines which show the effect of adding $A_{v}=8^{m}$ of extinction to the two starburst tracks. However, the radio emission from these galaxies would then be undetectable at $z>2$ with current sensitivity.

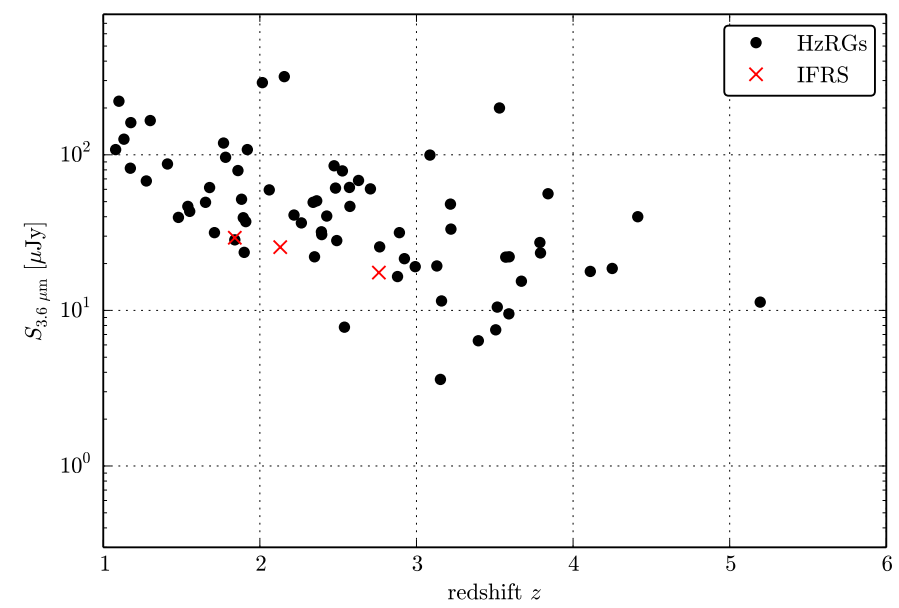

Fig. 3. 3.6 $\mu \mathrm{m}$ IR flux density versus redshift, adapted from Norris et al. (2011). Shown is the sample of HzRGs from Seymour et al. (2007) as black dots and the three IFRS whose redshifts are presented in this work as red crosses. The IFRS are located in the same parameter range as the HzRGs and seem to follow their $S_{3.6 \mu \mathrm{m}}-z$ relation. It appears that IFRS define the lower bound of HzRGs.

dwarf galaxies, and quasars. Furthermore, we took all available photometric data of the IFRS from Norris et al. (2006). These data consist of optical ( $G$ and $R$ bands), IR (3.6 $\mu \mathrm{m}$ and $4.5 \mu \mathrm{m})$, and radio $(1.4 \mathrm{GHz})$ flux densities for all three IFRS, while S212 and S265 provide more data points in the optical and IR range.

To model the SEDs of IFRS, we shifted the template SEDs to the rest-frame and scaled them in luminosity to match the observed $3.6 \mu \mathrm{m}$ flux density of the IFRS. Extinction was added

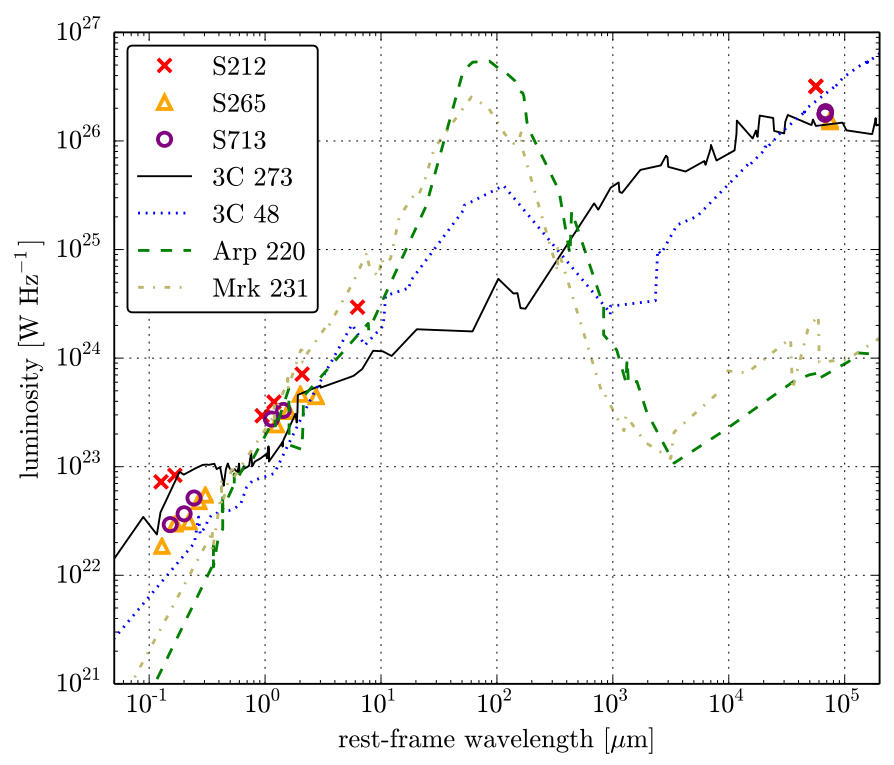

Fig. 4. Rest-frame SED modelling for the three IFRS whose redshifts were determined in this work. All templates are shifted to the rest-frame and scaled in luminosity to match the photometric data points shown by red crosses (S212), orange triangles (S265), and purple circles (S713). While the SED templates of star forming galaxy Arp 220 (green dashed line) and Seyfert galaxy Mrk 231 (olive dashed-dotted line) clearly disagree with the available photometric data, the templates of the radioloud quasar 3C 273 (black solid line) and the compact steep spectrum (CSS) source 3C 48 (blue dotted line) fulfill the requirements.

in the rest-frame optical and near-IR, following a Calzetti et al. (2000) reddening law, where required by the photometric data. Furthermore, all available photometric datapoints of the IFRS from NED were also shifted to the IFRS rest-frames and used to test the template SEDs for compatibility with these available constraints.

Figure 4 shows the SED modelling for the three IFRS. We find that S265 and S713 have very similar SEDs; Infrared-faint radio source $\mathrm{S} 212$ is also similar, except that it is a factor of 2 or 3 brighter than the other two at all wavelengths. These SEDs are consistent with either the $3 \mathrm{C} 273$ or the $3 \mathrm{C} 48$ templates. In our modelling, we scaled down these two templates in luminosity by wavelength-independent factors of 17 and 10, respectively, to match the observed $3.6 \mu \mathrm{m}$ flux densities. In the case of the templates of $3 \mathrm{C} 273$ and $3 \mathrm{C} 48$, no adding of extinction was needed to match the available flux densities.

Except for 3C 273 and 3C 48, none of the templates applied matches the SEDs of the IFRS, even with reddening applied. This result is in agreement with the finding that IFRS are highredshift radio-loud AGN as confirmed by the spectra shown in Fig. 1. Our SED modelling is inconsistent with the alternative interpretation of IFRS mentioned by Norris et al. (2011), explaining IFRS as AGN that undergo heavy dust extinction.

\subsection{Are IR-fainter IFRS at higher redshifts? Cosmological relevance of IFRS}

In Sect. 5.1, we showed that our results provide evidence for the similarity between IFRS and HzRGs. In particular, we found our IFRS to be in agreement with the redshift-3.6 $\mu$ m flux density relation determined for HzRGs by Seymour et al. (2007) in the redshift range probed by our sample. However, our sample was essentially determined by observational constraints, since fainter sources would have required unreasonably long integration times on the largest facilities. 
If we assume that IFRS follow the relation for at least somewhat higher redshifts, then most IFRS in the ATLAS fields would be at even higher redshifts, potentially reaching 5 or 6 , since the IFRS analysed in this paper are the optically and IR-brightest IFRS in the ATLAS fields (see Sect. 2). Whilst measuring such redshifts in the optical regime requires prohibitive amounts of observing time, the detection of molecular lines such as $\mathrm{CO}$ at radio frequencies might be much more efficient. The Atacama Large Millimeter Array (ALMA) is therefore the instrument of choice with which to test this hypothesis of an extension of the redshift $-3.6 \mu \mathrm{m}$ flux density relation towards higher redshifts.

We have shown that IFRS have similar properties to HzRGs, but they have a significantly higher sky density of a few per square degree. If the IR-fainter IFRS are indeed at even higher redshifts, the number of AGN in the early universe would be larger, resulting in even greater problems with structure formation and the growth of SMBHs shortly after the Big Bang (see Haiman 2013 for a recent review). This had already been emphasised by Zinn et al. (2011) under the assumption that IFRS are AGN driven and are located at redshifts of 3 . Since our evidence supports their premises, we conclude that IFRS are likely to be cosmologically relevant.

\section{Conclusions}

We present the first spectroscopic data of four IFRS in the ATLAS fields from the VLT FORS2 and determine the properties of IFRS.

- We determined the first redshifts of ATLAS-IFRS and find three IFRS at $z=1.84,2.13$, and 2.76, providing evidence of the suggested high redshifts of IFRS.

- Broad emission lines with line widths between $3300 \mathrm{~km} \mathrm{~s}^{-1}$ and $8700 \mathrm{~km} \mathrm{~s}^{-1}$ found in all spectra substantiate the claim that IFRS contain AGN.

- Using the redshifts measured in this work, we present the first SED modelling of IFRS based on redshifts and find the template SEDs of radio-loud quasars to agree with that of IFRS.

- IFRS have derived radio luminosities similar to those of HzRGs, providing further evidence of the similarity of IFRS and HzRGs.

- We test the hypothesis that IFRS follow the same correlation between $3.6 \mu \mathrm{m}$ flux density and redshift as HzRGs. Our findings support this hypothesis in the tested redshift range and increase the likelihood that IR-fainter IFRS are at even higher redshifts, potentially reaching 5 or 6 . Considering their sky density of a few per square degree, IFRS would significantly increase the number of AGN in the early Universe, leading to even more problems with structure formation and the growth of SMBHs shortly after the Big Bang.
Acknowledgements. We thank Peter-Christian Zinn for providing the basics of the code for the SED modelling. AH acknowledges funding from Bundesministerium für Wirtschaft und Technologie under the label 50 OR 1202. The author is responsible for the content of this publication. Based on observations made with ESO Telescopes at the La Silla Paranal Observatory under programme ID 087.B-0813(A). IRAF is distributed by the National Optical Astronomy Observatory, which is operated by the Association of Universities for Research in Astronomy (AURA) under cooperative agreement with the National Science Foundation. This research has made use of the NASA/IPAC Extragalactic Database (NED) which is operated by the Jet Propulsion Laboratory, California Institute of Technology, under contract with the National Aeronautics and Space Administration.

\section{References}

Appenzeller, I., Fricke, K., Fürtig, W., et al. 1998, The Messenger, 94, 1 Boutsia, K., Grazian, A., Giallongo, E., et al. 2011, ApJ, 736, 41 Calzetti, D., Armus, L., Bohlin, R. C., et al. 2000, ApJ, 533, 682 Collier, J. D., Banfield, J. K., Norris, R. P., et al. 2014, MNRAS, 439, 545

Elvis, M., Wilkes, B. J., McDowell, J. C., et al. 1994, ApJS, 95, 1 Fan, X., Strauss, M. A., Becker, R. H., et al. 2006, AJ, 132, 117 Fanaroff, B. L., \& Riley, J. M. 1974, MNRAS, 167, 31

Fazio, G. G., Hora, J. L., Allen, L. E., et al. 2004, ApJS, 154, 10 Garn, T., \& Alexander, P. 2008, MNRAS, 391, 1000

Giacconi, R., Zirm, A., Wang, J., et al. 2002, ApJS, 139, 369

Haiman, Z. 2013, Astrophys. Space Sci. Lib., eds. T. Wiklind, B. Mobasher, \& V. Bromm (Berlin Heidelberg: Springer-Verlag), 396, 293

Hopkins, P. F., Hernquist, L., Martini, P., et al. 2005, ApJ, 625, L71

Huynh, M. T., Norris, R. P., Siana, B., \& Middelberg, E. 2010, ApJ, 710, 698

Kimball, A. E., \& Ivezić, Ž. 2008, AJ, 136, 684

Lamastra, A., Menci, N., Maiolino, R., Fiore, F., \& Merloni, A. 2010, MNRAS, 405, 29

Lonsdale, C. J., Smith, H. E., Rowan-Robinson, M., et al. 2003, PASP, 115, 897

Luo, B., Bauer, F. E., Brandt, W. N., et al. 2008, ApJS, 179, 19

Mauduit, J.-C., Lacy, M., Farrah, D., et al. 2012, PASP, 124, 714

Middelberg, E., Norris, R. P., Cornwell, T. J., et al. 2008a, AJ, 135, 1276

Middelberg, E., Norris, R. P., Tingay, S., et al. 2008b, A\&A, 491, 435

Middelberg, E., Norris, R. P., Hales, C. A., et al. 2011, A\&A, 526, A8

Moretti, A., Campana, S., Lazzati, D., \& Tagliaferri, G. 2003, ApJ, 588, 696

Norris, R. P., Afonso, J., Appleton, P. N., et al. 2006, AJ, 132, 2409

Norris, R. P., Tingay, S., Phillips, C., et al. 2007, MNRAS, 378, 1434

Norris, R. P., Afonso, J., Cava, A., et al. 2011, ApJ, 736, 55

Puccetti, S., Fiore, F., D’Elia, V., et al. 2006, A\&A, 457, 501

Richards, G. T., Kruczek, N. E., Gallagher, S. C., et al. 2011, AJ, 141, 167

Rieke, G. H., Young, E. T., Engelbracht, C. W., et al. 2004, ApJS, 154, 25

Rieke, G. H., Alonso-Herrero, A., Weiner, B. J., et al. 2009, ApJ, 692, 556

Robertson, B. E., Ellis, R. S., Dunlop, J. S., McLure, R. J., \& Stark, D. P. 2010 Nature, 468, 49

Schinnerer, E., Smolčić, V., Carilli, C. L., et al. 2007, ApJS, 172, 46

Seymour, N., Stern, D., De Breuck, C., et al. 2007, ApJS, 171, 353

Singh, V., Beelen, A., Wadadekar, Y., et al. 2014, A\&A, in press DOI: $10.1051 / 0004-6361 / 201423644$

Tody, D. 1986, in Instrumentation in astronomy VI, ed. D. L. Crawford, SPIE Conf. Ser., 627, 733

Volonteri, M., \& Rees, M. J. 2005, ApJ, 633, 624

Willott, C. J., Rawlings, S., Jarvis, M. J., \& Blundell, K. M. 2003, MNRAS, 339, 173

Wright, E. L. 2006, PASP, 118, 1711

Wright, E. L., Eisenhardt, P. R. M., Mainzer, A. K., et al. 2010, AJ, 140, 1868

Zinn, P.-C., Middelberg, E., \& Ibar, E. 2011, A\&A, 531, A14 